\title{
Procedimientos Rituales en la Resolución de Ejercicios en Contexto Algebraico en Estudiantes de Profesorado de Matemática
}

\author{
Ritual Procedures while Solving Tasks in Algebraic Context in Pre-Service \\ Mathematics Teachers
}

\author{
Jimena Fernández* \\ Cristina Ochoviet ${ }^{* *}$
}

\begin{abstract}
Resumen
En una investigación sobre el sentido de los símbolos (ARCAVI, 1994, 2007) en estudiantes de último año de la carrera de Profesor de Matemática para la enseñanza media, en un instituto de formación docente de Uruguay, detectamos la presencia de procedimientos rituales (VINNER, 2000) en la resolución de tareas algebraicas. Durante el análisis de las respuestas al cuestionario que se aplicó, observamos que algunos de los estudiantes resolvían un mismo ejercicio por dos procedimientos distintos. Luego de obtener por el segundo procedimiento el mismo resultado que por el primero, daban su respuesta. Mostraremos algunos ejemplos de este fenómeno, veremos que la elección de un procedimiento ritual le da confianza al estudiante en que el trabajo realizado es adecuado, veremos que este fenómeno está vinculado a las prácticas de aula y alejado de un aprendizaje significativo del álgebra.
\end{abstract}

Palabras-clave: Álgebra. Formación de Profesores. Procedimiento Ritual. Sentido de los Símbolos.

\begin{abstract}
In a research about symbol sense (ARCAVI, 1994, 2007) in Pre-Service Mathematics Teachers that were studying the last year to become Mathematics Teachers, we detected the presence of ritual procedures (VINNER, 2000) while solving algebraic tasks. During the analysis of the answers to the applied questionnaire, we observed that some of the students solved the same exercise using two different procedures. After obtaining the same answer by the second method as the one obtained by the first method, they were ready to give an answer. We will show some examples of this phenomenon and notice that by using a ritual procedure the student feels confident that his/her resolution is right. Moreover, we will see this phenomenon is linked to classroom practices and does not reveal meaningful learning of algebra.
\end{abstract}

Keywords: Algebra. Pre-Service Mathematics Teachers. Ritual Procedure. Symbol Sense.

\footnotetext{
* Magíster en Enseñanza de las Ciencias Exactas y Naturales por la Universidad Nacional del Comahue (UNCOMA). Profesora de Matemática en el Consejo de Formación en Educación (CFE), Montevideo, Uruguay. Dirección Postal: Asilo 3255, Montevideo, Uruguay. E-mail: surrumbu @gmail.com

** Doctora en Matemática Educativa por el Centro de Investigación en Ciencia Aplicada y Tecnología Avanzada del Instituto Politécnico Nacional (CICATA-IPN). Profesora de Didáctica de la Matemática en el Consejo de Formación en Educación (CFE), Montevideo, Uruguay. Dirección Postal: Asilo 3255, Montevideo, Uruguay. Email: cristinaochoviet@gmail.com
} 


\section{Introducción}

En relación a lo que se espera de un profesor de matemática, la NCTM (2000) plantea que estos deben saber y comprender profundamente la matemática que están enseñando. Deben disponer de ese conocimiento de manera flexible en sus tareas de enseñanza. Es imprescindible que los profesores sean conscientes de que las decisiones didácticas que toman, tienen un gran impacto en la forma en que los estudiantes aprenden matemática. Agrega que una meta consiste en lograr que los profesores puedan generar entornos ricos de aprendizaje y, para cumplir este objetivo, los conocimientos y las destrezas matemáticas del profesor juegan un papel fundamental.

En este trabajo presentaremos algunos resultados de un estudio más amplio ${ }^{1}$ que abordó el análisis del desarrollo del sentido de los símbolos, desde la perspectiva de Arcavi (1994, 2007), en estudiantes del último año del profesorado de matemática. En el proceso de análisis de los resultados visualizamos un fenómeno que no habíamos previsto, pero que llamó mucho nuestra atención. Este fenómeno consistió en resolver un mismo ejercicio por dos procedimientos distintos. Recién al verificar, por el segundo procedimiento, que el resultado obtenido era el mismo que por el primer procedimiento, entonces el alumno se mostró dispuesto a dar su respuesta. Para comprender este fenómeno nos vimos llevadas a ampliar la perspectiva teórica y fue así que incluimos el punto de vista de Vinner (2000), y lo que él denomina procedimientos rituales. Esto nos permitió dar explicación al fenómeno detectado. En este trabajo nos enfocaremos, particularmente, sobre este último asunto.

\section{Las dificultades con los símbolos}

Las dificultades en relación al uso significativo de las letras están presentes tanto en estudiantes de enseñanza media (BILLS, 2001; SHARMA, 2000; PANIZZA; SADOVSKY; SESSA, 1996; WAGNER, 1983), como en estudiantes universitarios de distintas carreras vinculadas a la matemática en niveles iniciales y avanzados (ALURRALDE; IBARRA, 2008; URSINI; TRIGUEROS, 2006; TRIGUEROS; URSINI, 2003; MORALES; DÍAZ, 2003). Agregado a esto, podemos afirmar que los profesores de matemática en formación, y también los profesores experimentados, no son ajenos a estas dificultades (ACEVEDO; FALK, 2000;

\footnotetext{
${ }^{1}$ Fernández, J. (2013). El desarrollo del sentido de los símbolos en la formación inicial de profesores de matemática. Reflexiones en torno a la enseñanza del álgebra. Tesis de Maestría no publicada, Universidad Nacional del Comahue. Neuquén - Argentina.
} 
POPE; SHARMA, 2001; JUÁREZ, 2011; GONZÁLEZ; GONZÁLEZ, 2011). En Pope y Sharma (2001) se evidencia que los problemas referidos a la falta de sentido con que se trabaja en álgebra, están presentes en los docentes en formación y también en los profesores experimentados aunque en menor grado. Por ejemplo, hallaron que las raíces cuadradas y los inversos, al utilizarse como funciones aplicadas a variables, no son comprendidos adecuadamente por profesores experimentados, ni por profesores en formación. Juárez (2011) detectó que el uso de las letras en el que los profesores de matemática del nivel secundario muestran poseer un mejor entendimiento, es el de la variable como incógnita. Este autor reportó que los mejores resultados se obtuvieron en preguntas relacionadas a la manipulación, que es un aspecto en el que se enfatiza mucho en la enseñanza secundaria. Sin embargo, los profesores presentaron dificultades para encontrar patrones en secuencias y para utilizar una incógnita para resolver problemas que así lo requieren. Además, observó que los docentes tenían dificultades para relacionar lo aprendido en cursos de álgebra superior, propios de la formación de grado, con el álgebra que el docente debe enseñar en secundaria.

\section{El sentido de los símbolos}

El estudio que realizamos se propuso como objetivo, explorar y analizar el desarrollo del sentido de los símbolos que manifiestan en la resolución de diferentes situaciones donde lo algebraico está en juego, los estudiantes del cuarto y último año de la carrera de Profesor de Matemática para enseñanza secundaria en un instituto de formación docente del Uruguay.

Elegimos trabajar con el marco teórico desarrollado por Arcavi $(1994,2007)$ en relación al sentido de los símbolos, utilizando los comportamientos que él describe como indicadores del desarrollo del sentido de los símbolos.

En estos trabajos sobre el sentido de los símbolos, Arcavi plantea que es un complejo y multifacético sentimiento hacia los símbolos $\mathrm{y}$, para explicarlo, presenta distintos comportamientos que ilustran el significado que él atribuye a ese concepto. Los comportamientos que Arcavi describe como los componentes más importantes que demuestran haber construido un sentido de los símbolos son:

1. Amigabilidad con los símbolos. Esto incluye la comprensión de los símbolos de forma que estén fácilmente disponibles para ser usados cuando es conveniente y para ser dejados de lado en el caso en que sean una opción engorrosa.

2. Capacidad para manipular y también leer a través de expresiones simbólicas. Esto incluye la capacidad de adoptar una visión global de las expresiones simbólicas y, por otro 
lado, poder separarse de los significados para que las manipulaciones sean rápidas y eficientes. La lectura de y a través de las expresiones simbólicas con el objetivo de captar significados agrega niveles de conexión y razonabilidad a los resultados.

3. Tomar conciencia de que es posible diseñar exitosamente relaciones simbólicas que expresen cierta información dada o deseada.

4. Ser capaz de reconocer en expresiones simbólicas equivalentes, significados no equivalentes. La manipulación simbólica de las expresiones algebraicas nos permite obtener expresiones equivalentes, sin embargo cada expresión con la que nos enfrentamos puede ser fuente de nuevos significados.

5. La capacidad de seleccionar una representación simbólica y, en ciertos casos, reconocer nuestra propia insatisfacción con esa elección e ingeniárselas para buscar una mejor.

6. Realizar manipulaciones simbólicas guiadas por un objetivo buscado, evitando realizar operaciones circulares, y teniendo una visión global en la que se ven a los símbolos organizados de una determinada manera, y no solo como una concatenación de letras.

7. Conciencia de la necesidad de revisar los significados de los símbolos durante la aplicación de un procedimiento, durante la resolución de un problema, o durante la inspección de un resultado, y comparar esos significados con las intuiciones acerca de los resultados esperados y con la situación misma del problema.

8. Conciencia de que los símbolos pueden desempeñar roles distintos en distintos contextos, y desarrollar un sentido intuitivo de esas diferencias.

Con este marco, diseñamos un cuestionario con quince ejercicios. Cada uno de los ejercicios fue diseñado para poner en evidencia la aparición de uno o más de los comportamientos anteriormente descriptos.

\section{El contexto y las características de los estudiantes con los que trabajamos}

Como ya mencionamos anteriormente, centramos la atención en el desarrollo del sentido de los símbolos de los estudiantes del último año de la carrera de profesorado de matemática, en un instituto de formación de profesores del Uruguay. La carrera de profesor de matemática tiene una duración de cuatro años con un único plan de estudios de alcance nacional, durante los que los alumnos cursan asignaturas específicas de matemática (Álgebra, Análisis, Geometría, Geometría y Álgebra lineal, Probabilidad y Estadística etc.), asignaturas 
vinculadas a las Ciencias de la Educación (Pedagogía, Sociología de la educación, Psicología de la Educación etc.) y Didáctica de la Matemática con su respectiva práctica docente.

La formación de profesores en el Uruguay tiene la singular característica de ser acompañada por una práctica docente simultánea de tres años de duración en un aula de enseñanza secundaria, y no con posterioridad como en otras partes del mundo. Esta práctica se desarrolla de la siguiente manera. En el segundo y el tercer año de la carrera se asiste regularmente a un aula de matemática en enseñanza secundaria durante todo el año. El estudiante de formación docente observa las clases del profesor titular del grupo, planifica clases, dicta algunas clases - siempre en presencia del titular del grupo - y recibe una devolución de este acerca de su práctica, elabora pruebas, corrige tareas, siempre con la supervisión y acompañamiento del profesor titular del grupo. Tres veces al año recibe la visita de su Profesor de Didáctica del Instituto (que es con quien cursa la asignatura Didáctica de la Matemática) para observar su clase y realizarle una devolución cuyo objetivo es promover el análisis crítico de las prácticas y la formación de un profesional reflexivo.

El tercer año de práctica se cursa en el cuarto año de la carrera. En este caso, el estudiante que cursa la asignatura Didáctica de la Matemática, tiene un grupo a su cargo en un instituto de enseñanza secundaria de la educación pública uruguaya y debe cumplir con todas las responsabilidades de un docente: planificación y dictado de todas las clases del año, asistencia a reuniones de profesores y exámenes, elaboración y corrección de pruebas de evaluación, evaluación de los alumnos. Durante este proceso es acompañado y evaluado por su profesor de Didáctica de la Matemática que lo visita un mínimo de cinco veces al año. En esta visita se observa la clase que dicta el futuro profesor y una vez finalizada, se realiza el análisis detallado de la misma con el objetivo de ir obteniendo información que permita mejorar la práctica de la enseñanza.

Los estudiantes que participaron de nuestra investigación se encontraban cursando la Didáctica de la Matemática correspondiente al cuarto año de la carrera. Por lo tanto, todos ellos tenían un grupo a su cargo. En síntesis, se trabajó con toda la población de estudiantes, un total de dieciséis alumnos, del último año de la carrera de profesor de matemática que tenían grupo a su cargo en la enseñanza secundaria.

\section{Aplicación del cuestionario}

Se solicitó autorización a los profesores de Didáctica de los estudiantes para asistir a sus clases para la aplicación del cuestionario, a lo que ellos accedieron muy amablemente, por 
lo que fue realizado en el horario de clase. Los estudiantes resolvieron el cuestionario de quince ejercicios en forma individual, sin realizar consultas, y dispusieron de todo el tiempo que necesitaron, que fue aproximadamente de 90 minutos. Los ejercicios del cuestionario se les fueron entregando en grupos de a cinco (ver Anexo), debiendo entregar cada grupo luego de finalizado sin poder volver a ellos para realizar correcciones luego de que fueron entregados. El objetivo de esto fue que no modificaran su respuesta inicial en el caso en que el cuestionario generara aprendizaje durante su resolución. Todos los estudiantes que respondieron el cuestionario se mostraron interesados por la tarea a realizar, y muy motivados por la misma.

Los conocimientos relevantes para resolver los ejercicios del cuestionario son los adquiridos en los dos primeros años de la formación como profesor. Consideramos que las habilidades algebraicas continúan desarrollándose a medida que los individuos continúan estudiando matemática. Es decir, los contenidos matemáticos para enfrentar las actividades no corresponden a ningún curso en particular del profesorado, sino que se trata de conocimientos que no superan lo aprendido en el segundo año del profesorado y requieren de habilidades que se van adquiriendo en el proceso de estudio. En general, podemos decir que las actividades son accesibles a un estudiante que haya finalizado sus estudios de bachillerato con orientación en matemática o similar, por lo que, desde el punto de vista matemático, no requieren de herramientas matemáticas potentes, sino que más que nada demandan una buena interpretación de los símbolos y sentidos puestos en juego.

\section{Los procedimientos rituales}

Fue en el análisis de las producciones de los estudiantes, al momento de interpretar qué comportamientos descriptos por Arcavi $(1994,2007)$ se ponían en evidencia, que apareció un fenómeno que consideramos necesario explicar. Este fenómeno consistía, como ya señalamos anteriormente, en resolver un mismo ejercicio por dos procedimientos distintos. Recién al verificar, por el segundo procedimiento, que el resultado obtenido era el mismo que por el primer procedimiento, entonces el alumno estaba dispuesto a dar su respuesta. Si bien los comportamientos evidenciados en la resolución de los ejercicios eran enmarcables en la propuesta de Arcavi, quisimos dar una mirada más profunda a este proceso de resolución reiterada y sucesiva que presentaron algunos estudiantes de profesorado. 
Por este motivo, decidimos complementar el marco teórico de nuestro trabajo con la noción de procedimiento ritual, desarrollada por Shlomo Vinner en sus trabajos de los años 1997 y 2000, que, entendimos, permitía explicar el fenómeno que habíamos detectado.

Vinner (2000) traslada la idea cotidiana de comportamiento ritual, específicamente en lo relacionado con la religión, a la educación matemática. Él dice que es posible interpretar el comportamiento de una persona en una situación dada, a través del análisis de su sistema de creencias al respecto de esa determinada situación. Esto es, cómo ve la persona esa situación, qué piensa que esa situación es, qué espera de esa situación, cómo piensa que debe actuar frente a esa situación etc. Dice que todas las personas tenemos creencias al respecto de las situaciones en que participamos. Por ello, sugiere que existe una necesidad de creencias de todo tipo en los seres humanos. A partir de esto es que enfoca su mirada en el dominio en el que, según él, las creencias tienen un papel preponderante: la religión. La religión no es únicamente la creencia en un determinado Dios, sino que incluye instrucciones acerca de cómo se debe actuar con otros seres humanos y con Dios. Son estas instrucciones las que dan lugar a los rituales religiosos.

Pero además de los rituales religiosos, los seres humanos estamos constantemente cumpliendo con rituales seculares, es decir, actos que realizamos de forma tradicional que forman parte de nuestra identidad como grupo. Por ejemplo, darnos la mano como forma de saludo, celebrar cumpleaños, firmar contratos, sentarnos alrededor de la mesa de una determinada manera etc.

Teniendo en cuenta todo esto, es que Vinner (2000) afirma que los rituales tienen algunas funciones centrales en la vida humana. Para este autor, existen dos puntos básicos que relacionan los rituales con las necesidades humanas: el cumplimiento de un ritual proporciona seguridad al individuo que lo cumple y ayuda a la persona a desarrollar su identidad social, su identidad nacional, así como otro tipo de identidades, que constituyen una necesidad básica del ser humano. Entonces, según él, es posible afirmar, también, que los rituales son una necesidad psicológica de los seres humanos y que la mente humana ha desarrollado esquemas mentales especiales para hacer frente a los rituales. Estos esquemas identifican rituales y producen comportamiento ritual. Son llamados esquemas rituales. Si una persona cree que se encuentra en una situación ritual, entonces actuará en esa situación, guiada por el esquema ritual que está asociado a ella.

De todo esto se desprende que los rituales se formarán y se llevarán a cabo en todo ámbito de la vida de las personas, incluso en el educativo y, en particular, en la educación matemática. Centrándose en el ámbito educativo, Vinner (2000) define un procedimiento 
ritual a partir de la comparación con ciertas características de los rituales religiosos. Para él es posible identificar un procedimiento como ritual en la educación matemática, si nos enfrentamos a una secuencia de palabras (o símbolos matemáticos) y acciones (o acciones matemáticas) en las que observemos algunas de esas características. Por un lado, la persona que cumple con dicha secuencia de símbolos o acciones matemáticas considera que, al cumplirla, complacerá a alguien. Complacerá al sistema, a los profesores o maestros, a los padres o quizás, también, se complacerá a sí misma. En el procedimiento seleccionado subyace la idea de que es ese y no otro, el procedimiento con el que logrará satisfacer a quien juzgue su trabajo. Por otro lado, es posible que la persona que lleva adelante esa secuencia de acciones o palabras no comprenda por qué es necesario llevar a cabo tal secuencia, pero igualmente la realiza. Por último, el individuo que realiza el procedimiento ritual considera que seguir esa determinada secuencia de palabras o acciones dará aprobación a ciertos aspectos involucrados en el ritual, que no son necesariamente claros para el individuo durante el cumplimiento del ritual.

Agrega que en la actividad matemática existe una gran cantidad de procedimientos que utilizamos para resolver determinadas tareas y que es posible pensar que los estudiantes de matemática llevan a cabo estos procedimientos de la misma manera que las personas llevan a cabo rituales seculares o religiosos. Algunas actividades muy comunes en matemática, como simplificar términos algebraicos, resolver ecuaciones, reducir a común denominador, trasponer términos, derivar e integrar, pueden ser vistas por los estudiantes como procedimientos rituales.

Según Vinner (2000) los esquemas rituales guían al individuo para llevar a cabo los rituales y afirma que en muchos estudiantes los esquemas rituales son activados al trabajar en matemática. Generalmente, cuando en un estudiante es activado un esquema ritual, el estudiante no es consciente de ello. Cuando se responde, quizás inconscientemente, con una reacción ritual frente a un determinado estímulo, la persona puede estar excluyendo, rechazando o inhibiendo otro tipo de reacción, la reacción significativa. Es aquí que el comportamiento ritual entra en contradicción con un aspecto fundamental de la educación matemática, el aprendizaje significativo. Vinner (2000) utiliza el adjetivo significativo para referirse a un aprendizaje que para el alumno tiene sentido. Esto significa que el estudiante puede razonar sobre lo que hace, explicar por qué lo hace y establecer relaciones con otros conceptos. Agrega que en educación matemática es frecuente hablar de aprendizaje significativo, pero no siempre utilizando esta denominación, y ejemplifica con Ausubel (1968), Skemp (1976) y Davis (1984). Finalmente, agrega que si consideramos a este tipo de 
aprendizaje como el principal objetivo de la educación matemática, es necesario que los docentes enfoquemos nuestros esfuerzos en hacer que nuestros estudiantes dejen de lado los procedimientos rituales para pasar a realizar acciones significativas. En este caso, Vinner reconoce que estaríamos llevando a nuestros estudiantes a actuar en contra de su naturaleza. Sin embargo, él aclara que de eso se trata la educación, de recomendaciones para actuar en contra de nuestra naturaleza y, en definitiva, esto es un aspecto fundamental de la cultura humana.

\section{Los procedimientos rituales en las respuestas de los futuros profesores}

Al analizar los cuestionarios de los estudiantes, pudimos reconocer respuestas que enmarcamos dentro de la noción de procedimiento ritual de Vinner (2000). Observamos que algunos estudiantes realizaban dos procedimientos diferentes para resolver un mismo ejercicio del cuestionario. Es decir, a pesar de haber hallado la respuesta mediante un primer procedimiento que podríamos calificar como no habitual o no estándar, realizaban, a continuación, otro procedimiento más del tipo estándar - por ejemplo los procedimientos bien conocidos para extraer común denominador para resolver una ecuación racional - y llegaban nuevamente a la solución arribada por el primer procedimiento. Esta situación se presentó en las respuestas de cinco estudiantes diferentes, en ejercicios relativos a la resolución de ecuaciones racionales y a la resolución de sistemas de ecuaciones.

En los ejercicios que requerían la resolución de ecuaciones racionales, los estudiantes comenzaron por realizar un primer procedimiento guiado por la inspección de la expresión simbólica (Comportamiento 2 de Arcavi (1994, 2007)). Esta inspección permitía reconocer la raíz de la ecuación al identificar que el numerador es el triple del denominador. Sin embargo, luego de llegar a la solución por este procedimiento, los estudiantes realizaron un nuevo procedimiento realizando transformaciones algebraicas, como común denominador y trasposición de términos, para llegar a la misma solución ya encontrada por el primer procedimiento descripto. Veamos algunos ejemplos (Figura 1). 


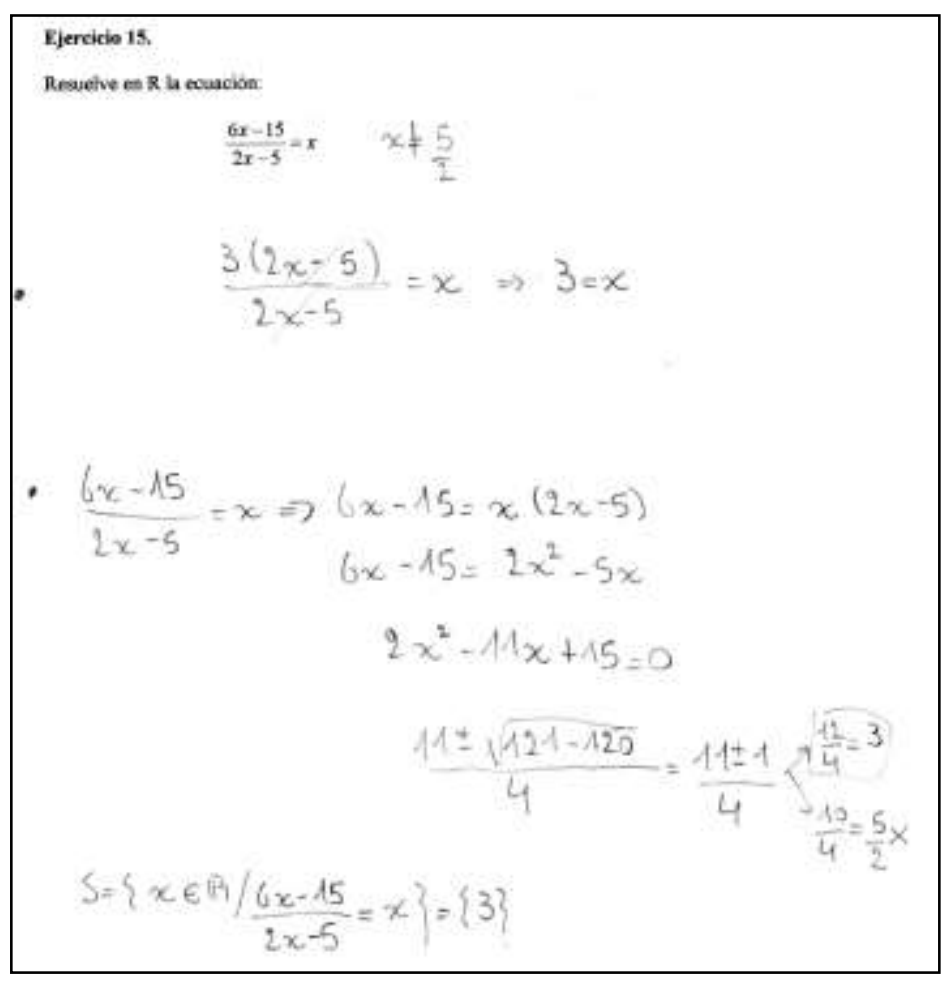

Figura 1 - Trabajo del Estudiante 2

El Estudiante 2 plantea dos resoluciones diferentes para la ecuación. En la primera realiza una inspección de la expresión planteada que lo lleva a realizar transformaciones algebraicas que le permiten evidenciar que $x=3$. Sin embargo, a continuación, plantea otra resolución, utilizando un procedimiento algebraico estándar, que le permite corroborar que la solución a la que arribó en primera instancia es la correcta. Este segundo procedimiento, podemos ubicarlo dentro de lo ritual. Pensamos que el estudiante siente la necesidad de realizar un segundo procedimiento guiado por la necesidad de complacer a quien observe sus respuestas en el cuestionario. Hablamos de necesidad de complacer, en tanto el segundo procedimiento se encuadra en los procedimientos habituales de resolución de una ecuación como la dada. Este segundo procedimiento, además de permitirle al estudiante corroborar el resultado hallado por el primer procedimiento, le da la confianza de que el resultado hallado previamente por un procedimiento no estándar, es correcto. Valida la solución hallada al cumplir con una determinada secuencia de acciones.

Esta necesidad del estudiante, nos muestra una escasez de confianza en el primer procedimiento realizado. Pensamos que no confía en la lectura que realiza de los símbolos.

A continuación, presentamos el trabajo del estudiante 8 (Figura 2): 


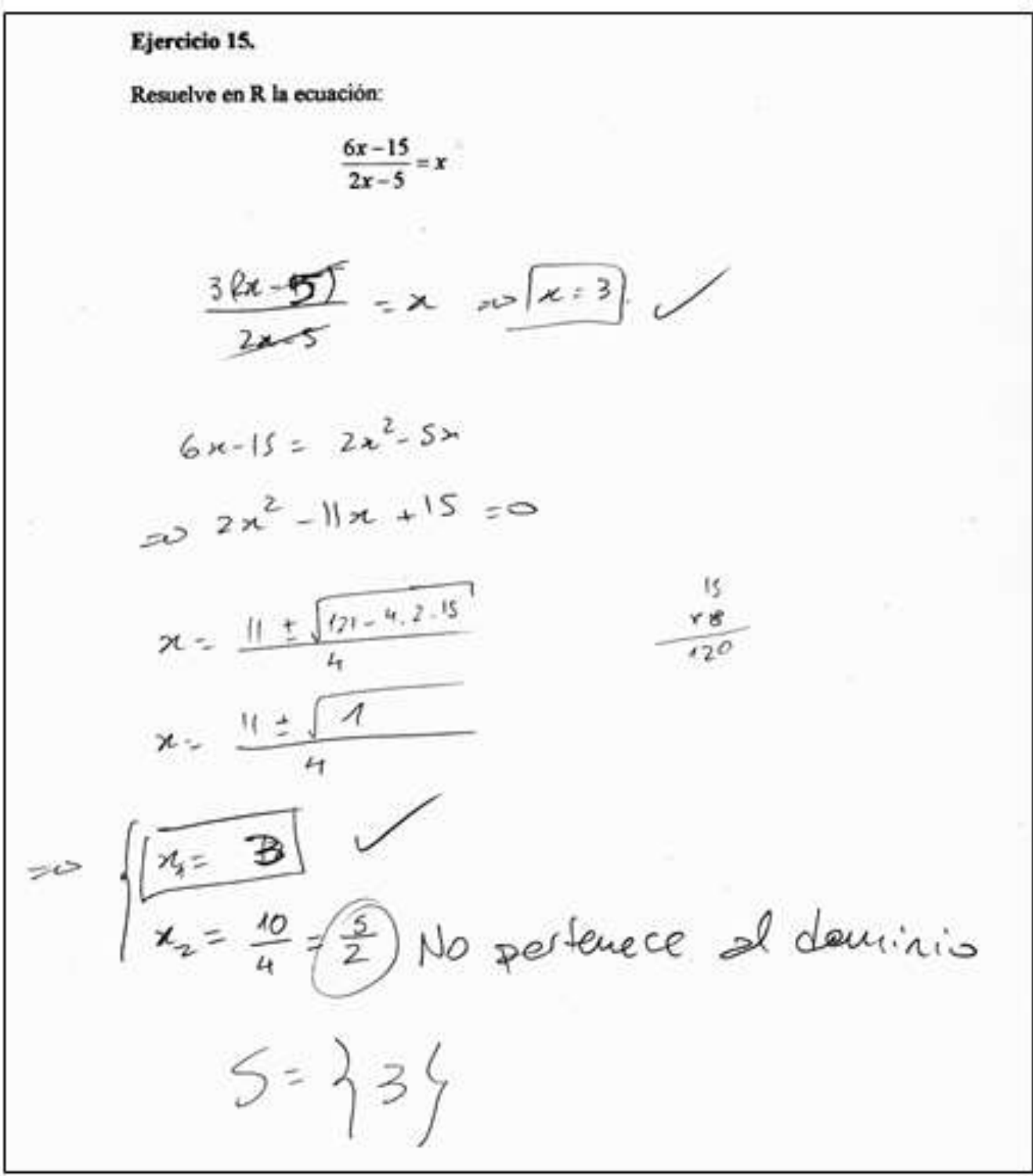

Figura 2 -Trabajo del Estudiante 8

En este caso, también creemos que el Estudiante 8 no confía en su primer procedimiento. No está seguro de que la solución hallada de esa manera sea correcta o no confía en que quien vea su trabajo acepte esa forma de resolver la ecuación. Por esto, el estudiante se ve llevado a plantear un segundo procedimiento para resolver la ecuación. Es este segundo intento el que daría cuenta de la presencia de un procedimiento ritual. El estudiante considera que debe cumplir con una determinada secuencia de acciones matemáticas más del tipo estándar, como transformaciones algebraicas y trasposición de términos, obtención de una ecuación de segundo grado que resuelve con la conocida fórmula resolvente. Estas acciones le proporcionan la confianza de que de esa manera arribará a la solución correcta dado que es una secuencia de trabajo bien conocida por él, por encuadrarse en el formato estándar para resolver una ecuación racional. El cumplir la segunda secuencia 
de acciones, le asegura que la solución a la que arriba es la solución correcta, mientras que parecería no estar seguro de esto luego de aplicar el primer procedimiento.

En los ejercicios en los que se pretendía que los estudiantes hallaran la cantidad de soluciones de un sistema de ecuaciones (y no las soluciones), observamos que los estudiantes realizaron la representación gráfica asociada a las ecuaciones del sistema para luego identificar los puntos de intersección de los gráficos en caso de que existieran. Sin embargo, vimos que luego de esto agregaron un procedimiento analítico para resolver el sistema con el objetivo de hallar analíticamente los pares ordenados que son soluciones comunes a ambas ecuaciones del sistema (que por cierto no se pedía). Vemos algunos ejemplos a continuación (Figura 3).

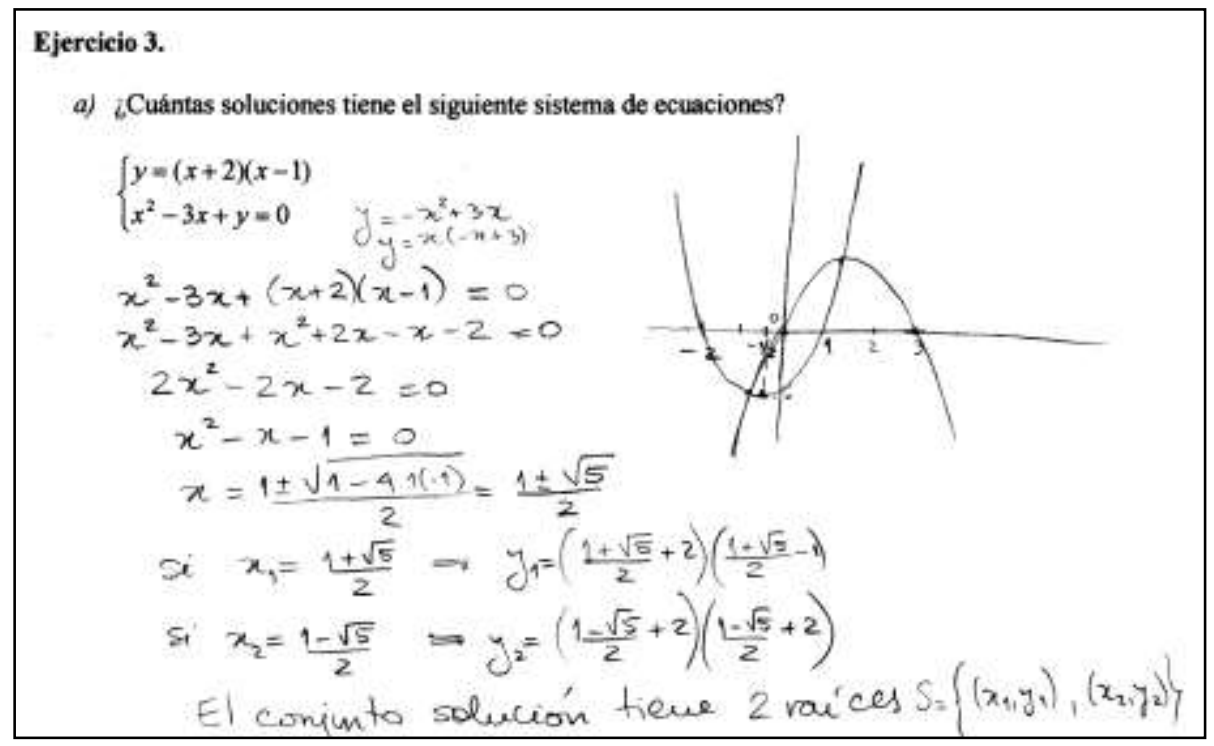

Figura 3 -Trabajo del Estudiante 13

En primer lugar es importante notar que la consigna pide averiguar cuántas soluciones tiene el sistema y no cuáles son. En la resolución que da el Estudiante 13 para la primera parte, aparecen dos formas de hallar la cantidad de soluciones del sistema. Por un lado, realiza la representación gráfica de las ecuaciones del sistema y, por otro, aplica un procedimiento analítico. Por ambos caminos logra hallar la cantidad de soluciones correctamente. Pensamos que es posible que el estudiante haya sentido la necesidad de comprobar, analíticamente, la respuesta hallada gráficamente. Esto nos indica que no confía en la resolución gráfica. Es posible que el trabajo analítico se esté realizando como un ritual por parte del alumno, quizás le brinda más seguridad respecto del abordaje gráfico realizado, o tal vez crea que con el procedimiento algebraico complacerá a quien corrija el ejercicio, dado que procede aplicando un método habitual en las aulas como es el de sustitución. Además, vemos que halla las coordenadas de los puntos de intersección aunque esto no es solicitado en el ejercicio. Esto da 
fuerza a la idea de que el trabajo analítico funcionó a manera de ritual, ya que el estudiante necesita atravesar la secuencia de símbolos presentada para poder dar respuesta (dos raíces) a la situación planteada.

Vemos, a continuación, otro caso similar al descripto anteriormente (Figura 4).

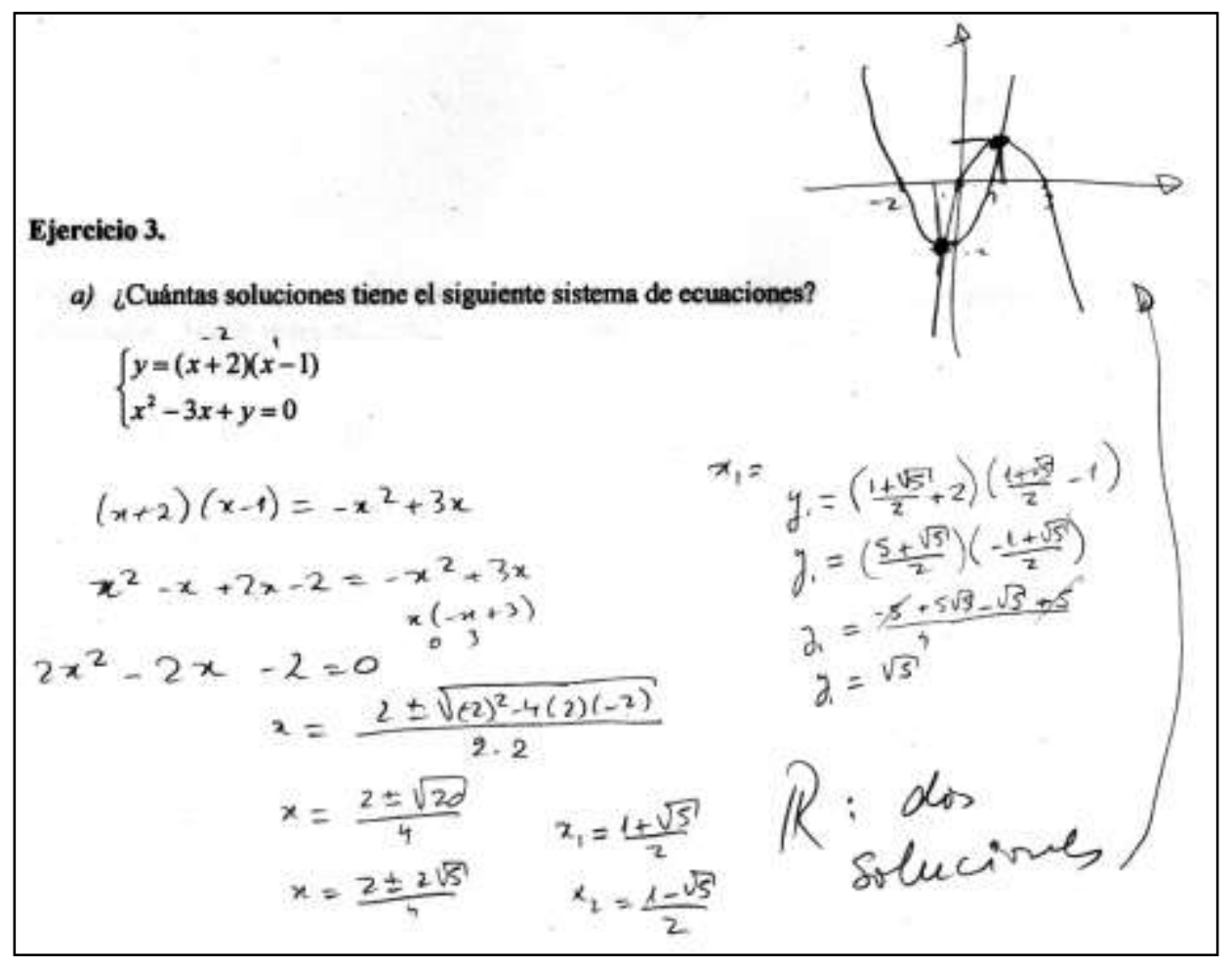

Figura 4 - Trabajo del Estudiante 9

El Estudiante 9 realiza dos procedimientos al intentar resolver el sistema de ecuaciones. Por un lado, realiza las representaciones gráficas de las ecuaciones del sistema y observa que existen dos puntos de intersección. Por otro, despeja y de la segunda ecuación y la sustituye en la primera. De esta manera halla dos valores posibles para la $x$ y luego sustituye en la primera ecuación con el objetivo de hallar los correspondientes valores de $y$, aunque no concluye este trabajo. No obstante, parecería que lo hecho le alcanza para estar seguro de que el sistema tiene dos soluciones. Es decir, el procedimiento analítico funciona como comprobación del método gráfico que utilizó previamente. Así lo permite afirmar la flecha que dibuja, que sale de la frase dos soluciones y apunta a la gráfica. Esto nos muestra que, para este estudiante, el procedimiento algebraico confirma el procedimiento gráfico.

Entendemos que el procedimiento analítico funcionó a manera de ritual, ya que la secuencia de símbolos que utiliza y las acciones algebraicas que realiza nos sugieren que confía en que, a través de este trabajo, obtendrá el resultado buscado, y necesita transitarlo a pesar de que no le sea de utilidad dada la claridad de las gráficas obtenidas. También 
observamos que las secuencias algebraicas utilizadas por el estudiante están presentadas de una forma que da aprobación a ciertos aspectos involucrados en el ritual que no son necesariamente claros durante el ritual. Por ejemplo, el hecho de hallar el valor de $y$ correspondiente al valor de $x_{1}$, no viene al caso para dar respuesta a la pregunta, no obstante es, para el estudiante, parte de la secuencia de acciones que opta por realizar.

Pensamos que el procedimiento algebraico estándar realizado por los estudiantes para resolver un sistema de ecuaciones, cuando lo que se preguntaba era cuántas soluciones tenía el sistema y no cuáles, puede entenderse como un procedimiento ritual. Los estudiantes no confían en la solución hallada por el primer procedimiento de naturaleza gráfica y sienten la necesidad de realizar un segundo procedimiento (el algebraico) en el que sí confían.

El segundo procedimiento realizado, de naturaleza algebraica, es el procedimiento que se realiza habitualmente en las clases de matemática, ya que es la resolución que los profesores suelen enseñar tanto en la enseñanza secundaria como en el nivel terciario. Es por esto que consideramos que responde a la necesidad del estudiante de cumplir con una determinada secuencia de acciones con la que pueda complacer ya sea a quien revise el ejercicio o, también, a sí mismo, dado que es un procedimiento que se lo reconoce como eficaz al haber sido objeto de enseñanza en el aula. Además, creemos que es posible que los estudiantes no confíen en la solución hallada por el primer procedimiento de naturaleza gráfica y se aseguren de que está bien resuelto, luego de atravesar el ritual y arribar a la misma respuesta. En los casos planteados, este procedimiento era completamente prescindible, por esto lo identificamos como ritual.

En los cuestionarios de la mayoría de los estudiantes pudimos evidenciar respuestas en las que el procedimiento realizado es el procedimiento habitual o estándar en las clases de matemática. En muchas oportunidades, claro está que, dependiendo del problema, este procedimiento obliga al estudiante a utilizar herramientas que resultan, muchas veces, más potentes de lo necesario para resolver la situación y en algunas oportunidades, son innecesarias. Pensamos que es posible interpretar estas elecciones de los estudiantes como respuestas rituales a un determinado estímulo. Por ejemplo, en los ejercicios en los que se debe resolver una ecuación racional, los estudiantes realizan en la mayoría de los casos común denominador y trasponen términos aunque es posible resolver las ecuaciones de una manera más sencilla, a través de la inspección de las expresiones. Es posible que el estímulo provocado por este tipo de ecuaciones, provoque una reacción ritual en los estudiantes que los lleva a optar por este tipo de resolución dejando de lado, quizás inconscientemente, otro tipo de abordajes. 
Pensamos que algo similar sucede en las respuestas en que los estudiantes deben hallar la cantidad de soluciones para un determinado sistema de ecuaciones. En ese caso, los estudiantes optan por hallar las coordenadas de los puntos de intersección de manera analítica en la mitad de los cuestionarios (sin considerar los casos en los que hacen dos procedimientos). Aunque no está solicitado en la pregunta, muchos de estos estudiantes, luego de reconocer que hay dos soluciones, intentan hallar las coordenadas de estos puntos. Creemos que es posible interpretar como un ritual este procedimiento, ya que es habitual en las aulas que los ejercicios de este tipo pidan hallar el conjunto solución y no indicar solamente la cantidad de soluciones.

\section{Síntesis y problematización de las respuestas de los estudiantes con presencia de procedimientos rituales}

A continuación presentamos, a manera de síntesis, una tabla que permite observar los procedimientos rituales que pudimos evidenciar en las producciones de cada estudiante.

\begin{tabular}{|c|c|c|c|c|c|c|c|c|}
\hline & Couportamieato I & Congurtamiets 2 & Compertamieteto 3 & Compotamieto 4 & Comportamietio 5 & Conpurtamieta 6 & Comperstamimitn? & Compotaminto 8 \\
\hline \multicolumn{9}{|l|}{ Estudiante I } \\
\hline \multicolumn{9}{|l|}{ Estudiante 2} \\
\hline \multicolumn{9}{|l|}{ Estudiante 3} \\
\hline \multicolumn{9}{|l|}{ Estudiante 4} \\
\hline \multicolumn{9}{|l|}{ Estudiante 5} \\
\hline \multicolumn{9}{|l|}{ Estudiante 6} \\
\hline \multicolumn{9}{|l|}{ Estudiante 7} \\
\hline \multicolumn{9}{|l|}{ Estudiante 8} \\
\hline \multicolumn{9}{|l|}{ Estudiante 9} \\
\hline \multicolumn{9}{|l|}{ Estudiante 10} \\
\hline \multicolumn{9}{|l|}{ Estudinate II } \\
\hline \multicolumn{9}{|l|}{ Estudiante 12} \\
\hline \multicolumn{9}{|l|}{ Estudinate 13} \\
\hline \multicolumn{9}{|l|}{ Estudinnte 14} \\
\hline \multicolumn{9}{|l|}{ Estudiante 15} \\
\hline Estudinate 16 & & & & & & & & \\
\hline
\end{tabular}

Presencia de procedimientos rituales

Presencia de procedimientos que podrian ser rituales.

Figura 5 - Matriz que muestra en forma sintética la presencia de procedimientos rituales de Vinner (2000) asociados a los comportamientos de Arcavi (1994, 2007)

Los procedimientos rituales (regiones gris oscuro en la Figura 5) se mostraron con mayor fuerza asociados a los comportamientos 1 y 2 de Arcavi (1994, 2007), pero solamente se hicieron presentes en aquellos ejercicios que solicitaban resolver ecuaciones racionales con una variable o sistemas de ecuaciones con dos variables. Esto nos hace pensar que fueron las 
características de los ejercicios que diseñamos para observar tales comportamientos, las que favorecieron la emergencia de procedimientos rituales. Esta conjetura es razonable, ya que en la educación matemática, tanto del nivel secundario como del nivel terciario, se realiza un fuerte énfasis en la enseñanza de procedimientos estándar tanto para resolver ecuaciones racionales como es el conocido método que consiste en reducir a común denominador ambos miembros de la expresión o como lo son los métodos algebraicos de sustitución o igualación para resolver un sistema de ecuaciones con dos variables. El ejercicio de la docencia requiere la capacidad de poder representar el conocimiento de distintas maneras, de seguir el razonamiento implicado en un procedimiento realizado por los estudiantes y de poder explicar un concepto de más de una forma (BALL, 2003; BALL; THAMES; PHELPS, 2008). Como estas capacidades son jerarquizadas en la práctica docente, sería posible pensar que los entrevistados quisieron mostrar que podían resolver una misma tarea de dos formas diferentes. No obstante, no nos inclinamos por esta interpretación dado que la presentación o búsqueda de dos resoluciones no aparece como una característica que atraviesa la resolución de todo el cuestionario sino que aparece puntualmente en algunos ejercicios $\mathrm{y}$, fundamentalmente, en aquellos para los cuales existe un procedimiento estándar bien conocido y disponible en el nivel que cursan los estudiantes.

Los procedimientos que podrían ser considerados como respuestas rituales (regiones gris claro en la Figura 5) refieren al empleo de herramientas matemáticas potentes para la resolución de situaciones problemáticas en las que no es necesario recurrir a ellas, como por ejemplo, el siguiente caso (Figura 6). 


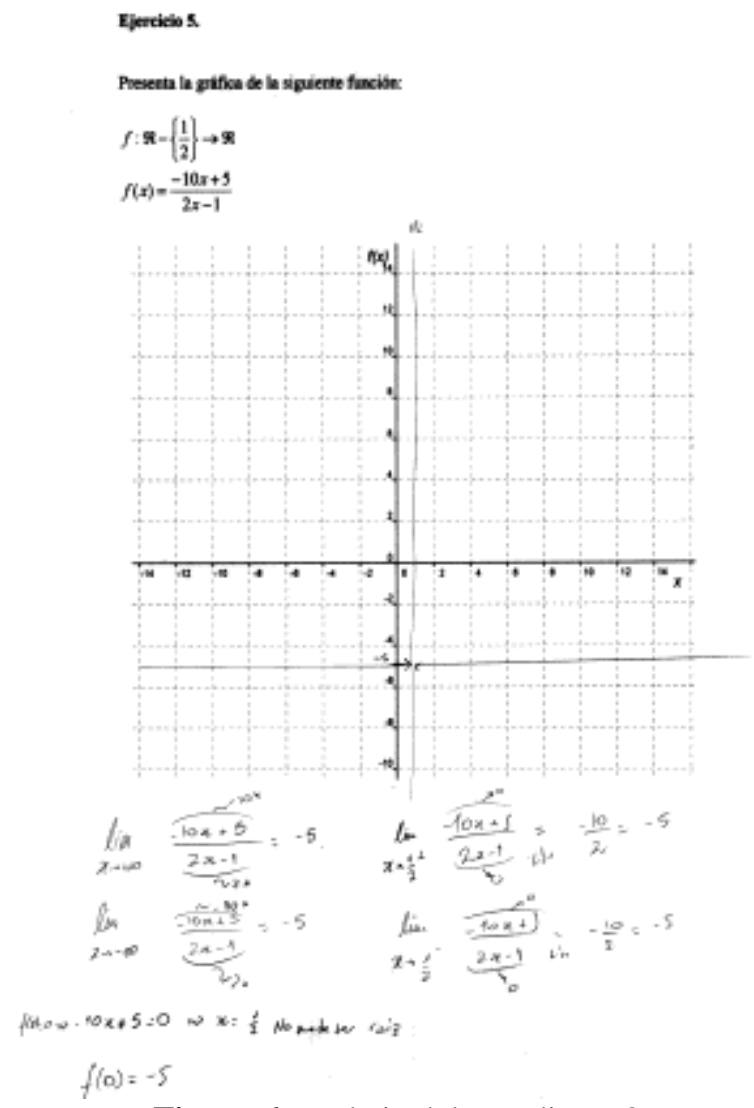

Figura 6 -Trabajo del Estudiante 8

El Estudiante 8 estudió los límites infinitos y los límites laterales en el valor de $x$ que no pertenece al dominio. También intentó hallar la raíz de la función, observó que no tiene, y halló la ordenada en el origen. El estudiante utilizó herramientas habituales y potentes en el contexto del análisis matemático, pero más bien útiles para la obtención de la gráfica de funciones de mayor complejidad. Una vez más, interpretamos que atravesar el ritual de este procedimiento le da al alumno la seguridad de que, por ese medio, obtiene la representación gráfica correcta. En este caso también inferimos que las características del ejercicio activaron procedimientos rutinarios que podrían ser entendidos como rituales en tanto no son para nada necesarios para responder el ejercicio. No obstante, más investigación sería necesaria al respecto, en tanto en este caso, no hay presencia de dos procedimientos distintos para resolver un mismo ejercicio, cómo sí aparece en los ejemplos presentados en la sección anterior.

\section{Conclusiones}

Fue posible evidenciar en los trabajos de los estudiantes procedimientos que pueden ser considerados como rituales en el sentido en que los identifica Vinner (2000). Como ya explicamos, un procedimiento es considerado ritual cuando la secuencia que lleva a cabo el 
individuo se selecciona bajo la idea de complacer a un tercero o a uno mismo con la seguridad de que, atravesando esa secuencia, se arribará a resultados correctos, aunque esta elección no es siempre consciente. Pensamos que en la elección de estos procedimientos, que como vimos no era necesario atravesar para responder, interviene la experiencia que tiene el estudiante con las prácticas que son de uso habitual en la enseñanza y, por lo tanto, aprobadas y eficaces en las aulas de matemática.

La elección de un procedimiento ritual le da confianza al estudiante en que el trabajo realizado es adecuado, y el cumplimiento de la secuencia de símbolos o acciones del ritual da validez a los diferentes aspectos del mismo, así como también a la solución arribada. Como se vio, en varios casos los estudiantes ya habían arribado a una respuesta correcta, pero no obstante, decidieron emprender una segunda resolución para afirmar la respuesta al ejercicio.

Pudimos ver esto claramente en los casos en los que los estudiantes presentaron dos procedimientos diferentes frente a un mismo ejercicio. En esos casos, a pesar de haber resuelto el ejercicio de una manera no estándar u original o poco común en las clases de matemática, realizaban a continuación un segundo procedimiento que podría considerarse más rutinario o estándar para cotejar si arribaban a la misma respuesta. Mostraron, de esta manera, una escasa confianza en aquellos procedimientos que se alejan de los que se utilizan habitualmente en las aulas. Este tipo de trabajo pudo verse, principalmente, en el trabajo con ecuaciones racionales y con sistemas de ecuaciones.

\section{Recomendaciones}

Es importante que los docentes de los futuros profesores de matemática tengan en cuenta que las prácticas que ellos valoran en sus clases tienen un fuerte impacto en el trabajo simbólico que realizan los estudiantes. Con el objetivo de evitar la realización de procedimientos rituales por parte de los estudiantes de profesorado de matemática, pensamos que, desde las aulas de formación docente, se debería estimular en los estudiantes la autonomía para confiar en procedimientos sui generis y no solamente en aquellos que forman parte del repertorio usual. Los estudiantes deberían poder ensayar con libertad diferentes abordajes de los problemas y ser capaz de distinguir los beneficios y dificultades de cada uno de ellos.

Si en las aulas de formación docente se trabaja con un único abordaje, brindado por el docente, es posible que el alumno realice este tipo de procedimiento a la hora de resolver una situación similar, generando, así, una respuesta ritual al problema. Es importante tener en 
cuenta, también, que en muchas oportunidades los estudiantes reaccionarán de forma inconsciente a un estímulo, ya que este activará un esquema ritual y provocará una reacción ritual por parte del estudiante. Creemos, entonces, que se deberían generar espacios de reflexión en las aulas donde el alumno - futuro profesor - pueda pensar en torno a sus elecciones metodológicas, los motivos que lo llevaron a elegirla y las implicancias de estas.

Para finalizar, recordemos que los estudiantes que participaron de esta investigación, como futuros docentes de matemática, serán los responsables de propiciar los primeros acercamientos al álgebra de muchos estudiantes de enseñanza secundaria. El desarrollo del sentido de los símbolos que construyan en su pasaje por la formación docente, tendrá fuerte incidencia en las primeras construcciones en torno al álgebra que realicen sus estudiantes. Por lo tanto, pensamos que al ocuparnos en mejorar el desarrollo del pensamiento algebraico de los futuros docentes de matemática, estamos, también, aportando a la mejora del aprendizaje de sus futuros alumnos. Es por esto que consideramos fundamental el prestar atención a la manera en que presentamos la matemática a los futuros docentes, ya que es seguro que los aprendizajes que logren repercutirán en las posibilidades que tendrán sus estudiantes de aprender significativamente la matemática.

\section{Bibliografía}

ACEVEDO, M.; FALK, M. Formación del pensamiento algebraico de los docentes. Revista Latinoamericana de Investigación en Matemática Educativa, México DF, v. 3, n. 3, p. 245-264. Noviembre. 2000.

ARCAVI, A. Symbol Sense: Informal Sense- making in Formal Mathematics. For the Learning of Mathematics, Vancouver, v. 14, n. 3, p. 24-35. Noviembre. 1994.

ARCAVI, A. El desarrollo y el uso del sentido de los símbolos. Conferencia realizada como Profesor visitante, CRICED, Tsukuba University- Japan. 2007. Disponible en: $<$ http://ebookbrowse.com/arcavi05-el-desarrollo-y-el-uso-del-sentido-de-los-simbolos-docd37871752>. Acceso en: 1 junio 2011.

ALURRALDE, F.; IBARRA, L. El uso de las letras en álgebra: Análisis de una evaluación de estudiantes de primer año de ingeniería. Revista de Educación Matemática. 2008. Disponible en: <http://www2.famaf.unc.edu.ar/rev_edu/documents/vol_22/pro_Alurralde_tra.pdf >. Acceso: 27 nov. 2014.

AUSUBEL, D. P. Educational Psychology: A Cognitive View. New York: Holt, Rinehart \& Winston. 1968.

BALL, D. L. What Mathematical Knowledge is Needed for Teaching Mathematics? 2003. Disponible en: <http://www.erusd.k12.ca.us/ProjectAlphaWeb/index_files/MP/BallMathSum mitFeb03.pdf >. Acceso en: 27 nov. 2014 
BALL, D. L.; THAMES, M. H.; PHELPS, G. Content knowledge for teaching: What makes it special? Journal of Teacher Education, v. 59 n. 5, p. 389-407. 2008. Disponible en: <http://www. math.ksu.edu/ bennett/onlinehw/qcenter/ballmkt.pdf>. Acceso en: 27 nov. 2014.

BILLS, L. Shifts in the meanings of literal symbols. En: International Conference for the Psycology of Mathematics Education, 25th, 2001, Países Bajos, proceedings... Países Bajos: Ultrech. 2001. p. 161168.

\section{CONFERENCE BOARD ON MATHEMATICAL SCIENCES (CBMS). The mathematical education of teachers. Washington, DC: Author. 2001.}

DAVIS, R. Learning Mathematics: The Cognitive Science Approach to Mathematics Education. London: Croom Helm. 1984.

FERNÁNDEZ, J. EI desarrollo del sentido de los símbolos en la formación inicial de profesores de matemática. Reflexiones en torno a la enseñanza del álgebra. 2013, 402 hojas. Tesis (Maestría en Enseñanza de las Ciencias Exactas y Naturales) - Facultad de Ingeniería de la Universidad Nacional del Comahue. Neuquén, Argentina. 2013.

GONZÁLEZ, A.; GONZÁLEZ, F. Exploración del pensamiento algebraico de profesores de Matemática en formación- "La prueba EVAPAL". Acta Scientiae, Canoas, v. 13, n. 1, p. 31- 54. 2011. Disponible en: <http://www.periodicos.ulbra.br/index.php/acta/article/viewFile /22/19>. Acceso en: 27 nov. 2014.

JUÁREZ, J. Dificultades en la interpretación del concepto de variable en profesores de matemática de secundaria: un análisis mediante el modelo 3UV. Números. Revista de didáctica de las matemáticas, La Laguna, v. 76, p. 83-103. Marzo. 2011.

MORALES PERAL, L.; DÍAZ GÓMEZ, J. Concepto de variable: Dificultades de su uso a nivel universitario. Mosaicos Matemáticos, Sonora, n. 11, p. 109-114. Diciembre. 2003. Disponible en: <http://semana.mat.uson.mx/ MemoriasXVII/XIII/lina.pdf>. Acceso en: 27 nov. 2014.

\section{NATIONAL COUNCIL OF TEACHERS OF MATHEMATICS. Principals and Standards for School Mathematics. USA: NCTM. 2000.}

PANIZZA, M.; SADOVSKY, P.; SESSA, C. The first algebraic learning: the failure of success. In: Conference of the International Group for the Psychology of Mathematics Education, 20th, 1996, Valencia, proceedings... Valencia: Puig, L; Gutiérrez, A (Eds). 1996, p.107-114.

POPE, S.; SHARMA, R. Symbol Sense: Teacher's and student's understanding. In: British Society for Reaserch into Learning Mathematics, 21, 2001, Southampton, proceedings... Southampton: Winter, J. (Ed.). 2001.

SHARMA, R. Researching Students' Symbol Sense. In: British Society for Research into Learning Mathematics, 20, 2000, Roehampton, proceedings... Roehampton: Rowland, T. (Ed.). 2000.

SKEMP, R. Relational Understanding and Instrumental Understanding. MathematicsTeaching, Derby, n. 77 , p. 20-26. Diciembre. 1976.

TRIGUEROS, M.; URSINI, S. Starting collage student's difficulties in working with different uses of variable. Research in Collegiate Mathematics Education V. CBMS Issues in Mathematics Education. American Mathematical Society, Providence, v. 5, p. 1-29. Octubre. 2003 
URSINI, S.; TRIGUEROS, M. ¿Mejora la comprensión del concepto de variable cuando los estudiantes cursan matemáticas avanzadas? Educación Matemática, México DF, v.18, n. 3, p. 5-38. Diciembre. 2006.

VINNER, S. The pseudo-conceptual and the pseudo-analytical thought processes in mathematics learning. Educational Studies in Mathematics, Países Bajos, v. 34, n. 2, p. 97-129. Noviembre. 1997.

VINNER, S. Mathematics Education- Procedures, Rituals and Man's Search for Meaning. Conferencia dictada en el ICME 9, Japón. 2000. Disponible en: <http://www.fisme.science.u u.nl/nwd/nwd2003/handouts/vinner.pdf >. Acceso en: 27 nov. 2014.

WAGNER, S. What are these things called variables? Mathematics Teacher, USA, v. 76, n. 1, p. 474-479. Octubre.1983

Submetido em Maio de 2014. Aprovado em Novembro de 2014. 


\begin{abstract}
ANEXO
A continuación presentamos el cuestionario aplicado y, en cada ejercicio, los comportamientos que son posibles de evidenciar.
\end{abstract}

\title{
Cuestionario
}

\section{Primer grupo de ejercicios}

\section{Ejercicio 1. (Comportamiento 1)}

a. Completa las celdas vacías para obtener un "cuadrado mágico" cuya constante es 30 (los números de cada fila, de cada columna o de cada diagonal, suman 30).

\begin{tabular}{|l|l|l|}
\hline & & \\
\hline & 10 & \\
\hline 4 & & 81 \\
\hline
\end{tabular}

b. Completa las celdas vacías para obtener un "cuadrado mágico" cuya constante es 15 (los números de cada fila, de cada columna o de cada diagonal, suman 15).

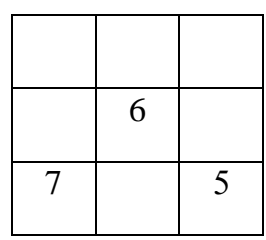

¿Qué observas? ¿Por qué sucede esto?

\section{Ejercicio 2. (Comportamiento 1 y 5)}

La intendencia quiere instalar una única hilera de 100 canteros hexagonales y rodearlos con baldosas también hexagonales según el siguiente modelo.

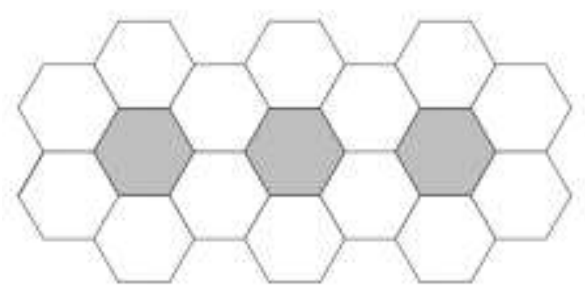

a. ¿Cuántas baldosas necesitará la intendencia?

b. ¿Cómo puedes calcular el número de baldosas necesarias para un número cualquiera de canteros? 


\section{Ejercicio 3. (Comportamiento 1 y 2)}

a. ¿Cuántas soluciones tiene en $\mathrm{R}$ el siguiente sistema de ecuaciones?

$\left\{\begin{array}{l}y=(x+2)(x-1) \\ x^{2}-3 x+y=0\end{array}\right.$

b. ¿Cuántas soluciones tiene en $\mathrm{R}$ el siguiente sistema de ecuaciones?

$\left\{\begin{array}{l}y=x^{2} \\ x=(y+1)(y+3)\end{array}\right.$

Ejercicio 4. (Comportamiento 2 y 5 )

Escribe una ecuación usando las variables E y P para representar la siguiente afirmación:

"Hay 6 veces más estudiantes que profesores en este instituto"

\section{Ejercicio 5. (Comportamiento 4)}

Presenta la gráfica de la siguiente función:

$$
\begin{aligned}
& f: \mathfrak{R}-\left\{\frac{1}{2}\right\} \rightarrow \mathfrak{R} \\
& f(x)=\frac{-10 x+5}{2 x-1}
\end{aligned}
$$

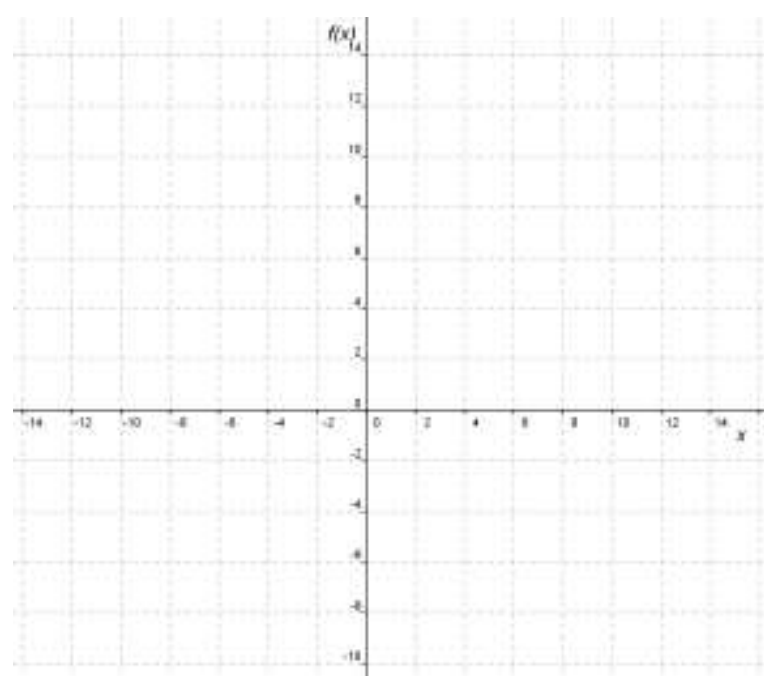

\section{Segundo grupo de ejercicios}

\section{Ejercicio 6. (Comportamiento 5)}

Elige un número impar, elévalo al cuadrado y réstale 1. ¿Qué puedes decir del resultado? 


\section{Ejercicio 7. (Comportamiento 3)}

La siguiente tabla de valores corresponde a una función $f$ de dominio real:

\begin{tabular}{|l|l|}
\hline$x$ & $f(x)$ \\
\hline-6 & -70 \\
\hline-5 & 0 \\
\hline-3 & 32 \\
\hline-1 & 0 \\
\hline 1 & 0 \\
\hline 2 & 42 \\
\hline
\end{tabular}

¿Puedes hallar una expresión analítica para dicha función? En caso afirmativo preséntala, en caso contrario indica por qué no es posible.

\section{Ejercicio 8. (Comportamiento 6 y 8 )}

Resuelve en R la siguiente ecuación de incógnita $x: x \sqrt{y}=1+2 x \sqrt{1+y}$

\section{Ejercicio 9. (Comportamiento 2)}

Resuelve en R: $\quad \frac{4 x+3}{2 x-5}+\frac{5}{2}=-\frac{3+4 x}{5-2 x}+x$

Ejercicio 10. (Comportamiento 6 y 7)

Observa el siguiente rectángulo:

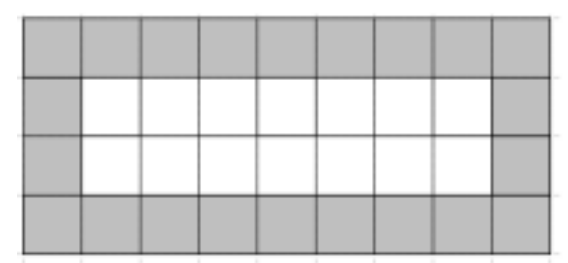

En este caso el número de celdas sombreadas en los bordes no es igual al número de celdas sin sombrear. ¿Es posible dibujar un rectángulo de forma que el borde sombreado (siempre de una celda de ancho) tenga el mismo número de celdas que el interior?

\section{Tercer grupo de ejercicios}

Ejercicio 11. (Comportamiento 8) 
Indica qué valor debe tomar a para que la siguiente expresión analítica corresponda a una función de primer grado.

$$
f(x)=(a x-a) x^{2}-3 x+4
$$

Ejercicio 12. (Comportamiento 2)

Resuelve en R:

$$
\frac{2 x+3}{4 x+6}=2
$$

Ejercicio 13. (Comportamiento 3)

La siguiente tabla de valores corresponde a una función $g$ de dominio real:

\begin{tabular}{|l|l|}
\hline$x$ & $g(x)$ \\
\hline 4 & 6 \\
\hline 3 & 0 \\
\hline 1 & 12 \\
\hline-1 & 16 \\
\hline-2 & 0 \\
\hline-3 & -36 \\
\hline
\end{tabular}

¿Puedes hallar una expresión analítica para dicha función? En caso afirmativo preséntala, en caso contrario indica por qué no es posible.

\section{Ejercicio 14. (Comportamiento 8)}

Indica qué valor debe tomar $d$ para que la siguiente expresión analítica corresponda a una función de primer grado.

$$
f(x)=\frac{x^{2}-4}{d-2}
$$

Ejercicio 15. (Comportamiento 2)

Resuelve en R:

$$
\frac{6 x-15}{2 x-5}=x
$$

